\title{
TRAYECTORIAS DISÍMILES Y PROYECTOS INDIVIDUALIZADOS: ORIGEN Y EXPERIENCIA EDUCATIVO-LABORAL DE LOS ESTUDIANTES DE CENTROS DE FORMACIÓN TÉCNICA
}

\section{Leandro Sepúlveda, Pamela Ugalde ${ }^{1}$}

\begin{abstract}
RESUMEN
El artículo presenta los principales resultados del estudio "Origen, expectativas y proyecto educativo-laboral de jóvenes estudiantes de Centros de Formación Técnica"2. La indagación consistió en la aplicación de un instrumento estructurado a una muestra de 901 estudiantes que estaban cursando el primer o segundo semestre de una carrera técnica en 23 CFT de la Región Metropolitana. El foco principal de este estudio era caracterizar el perfil y la trayectoria de los estudiantes de este sistema formativo, así como indagar en sus intereses y motivaciones personales.

Los principales resultados alcanzados dan cuenta de la heterogeneidad de la matrícula de este sistema formativo, la significativa presencia de estudiantes con trayectorias educativas y/o laborales previas, así como también el débil vínculo existente entre la formación técnica de nivel medio con la oferta de nivel superior.
\end{abstract}

Palabras clave: formación técnica, trayectoria educativo-laboral, educación vocacional

\section{DIFFERENT TRAJECTORIES AND INDIVIDUALIZED PROJECTS: ORIGIN AND EDUCATION/WORK EXPERIENCE OF STUDENTS AT TECHNICAL TRAINING CENTRES}

\begin{abstract}
The article presents the main results of the study, "Origin, expectations and labour education project of young students at Technical Training Centres"3. The research consisted in the application of a structured instrument to a sample of 901 students in their first or second semester of technical training at 23 TTCs in the Metropolitan Region. The main objective of this study was to characterize the profile and trajectory of students in this training system as well as discover their interests and personal motivations.

The main outcomes show the enrolment heterogeneity of this training system, the presence of students with previous work and/or educational experience, as well as a weak link between mid-level technical training and higher-level supply.
\end{abstract}

Key words: technical training, education/work trajectories, vocational education

1 Centro de Investigación y Desarrollo de la Educación, CIDE; Universidad Alberto Hurtado, Chile.Contacto: 1sepulve@cide.cl

2 Estudio realizado con el apoyo del Consejo Nacional de Educación entre los meses de septiembre de 2009 y junio de 2010

3 Undertaken with support of the National Education Council from September 2009 to June 2010. 


\section{TRAYECTORIAS DISÍMILES Y PROYECTOS INDIVIDUALIZADOS: ORIGEN Y EXPERIENCIA EDUCATIVO-LABORAL DE LOS ESTUDIANTES DE CENTROS DE FORMACIÓN TÉCNICA}

\section{Introducción}

La situación y perspectivas de desarrollo de la educación técnica superior en Chile constituyen ámbitos débilmente abordados por la investigación académica en los últimos años. El fuerte predominio alcanzado por la oferta de carreras universitarias en la formación de nivel superior ${ }^{4}$ y la, hasta ahora, escasa incidencia de las políticas públicas en el apoyo y sostenimiento del sector, constituyen factores que, entre otros, explican la situación de relativa marginalidad del debate sobre la educación técnica postsecundaria en general, y del sistema de centros de formación técnica, CFT, en particular.

Luego de un interés investigativo incipiente, contenido en una serie de publicaciones de fines de la década del 80 y principios de los 90 (entre otros, González, 1990; Pérsico, P. y Pérsico, M. C, 1995), la reflexión sobre la educación técnica ha estado restringida a dos ámbitos aunque relevantes, insuficientes: por una parte, el énfasis acerca del fomento del desarrollo productivo y la adecuación de capital humano funcional a esta tarea, y por otra, los ajustes institucionales que deberían ser necesarios para lograr consolidar una oferta formativa sistemática acorde a los desafíos del tiempo presente ${ }^{5}$. Esta línea de trabajo, de carácter fundamentalmente prescriptivo, no ha sido acompañada de información empírica consistente, sustentándose el debate, en muchas ocasiones, en supuestos no siempre verificables sobre el proceso de oferta/demanda de la formación técnica actual.

4 En el país actualmente existen 6,5 estudiantes en carreras universitarias por cada uno en carreras técnicas de nivel superior (Larrañaga, 2006).

5 Véase por ejemplo Informe Consejo de Innovación, Santiago, enero 2007. Disponible en: www.consejodeinnovacion.cl 
Los antecedentes disponibles ${ }^{6}$ señalan que en la actualidad el sistema de CFT constituye el principal ámbito de la formación técnicoprofesional en el país, acogiendo a 96.000 estudiantes, cifra que corresponde a casi el $60 \%$ de quienes han optado por esta modalidad de enseñanza de nivel superior. Sin embargo, la fuerte expansión de la matrícula de la educación terciaria en las últimas décadas (llegando a triplicarse el número total de estudiantes en los últimos 20 años) refleja un rezago general de la educación técnica superior en comparación con lo observado en la década de los 80. En efecto, entre 1990 y el año 2008, la proporción de estudiantes que cursaba alguna carrera al interior del sistema, frente al total de la educación superior, se redujo en 2,5 veces. Aunque esta situación se explica por la confluencia de diversos factores (ausencia de señales consistentes que orienten el mercado educativo para el desarrollo de ofertas funcionales a las necesidades del mercado del trabajo; débiles mecanismos de orientación vocacional para estudiantes y desregulación de la oferta formativa; crecimiento de expectativas sociales y mayor demanda por carreras de tipo universitarias entre los estudiantes y sus familias, entre otros), como consecuencia inmediata, la educación técnica ha tenido un lugar secundario en la discusión de políticas dirigidas hacia la educación superior, existiendo sólo en los últimos años una mayor preocupación y la emergencia de propuestas estratégicas orientadas a su desarrollo como parte de los desafíos de incremento de la capacidad de innovación productiva que requiere el país ${ }^{7}$.

En la actualidad, la oferta formativa nacional de los CFT se canaliza a través de 74 centros de formación que ofrecen más de 1.700 carreras de diverso tipo, particularmente en los sectores de administración y comercio, tecnología y salud. La matrícula se encuentra altamente segmentada por tipo de institución ${ }^{8}$ y la tasa

6 La información actualizada sobre el sistema de formación técnica de nivel superior se encuentra disponible en Consejo Nacional de Educación, http://www.cned.cl/y MINEDUC, http://www.divesup.cl/

7 Véase Bases para una Política de Formación Técnico-Profesional en Chile, Informe Ejecutivo. MINEDUC, Santiago 2009, disponible en: http://www.formaciontecnica.cl/archivosNoticias/ informe_bases.pdf; Villarroel, K./Secretaría Ejecutiva CNIC (2009).

8 Mientras que cuatro CFT concentran el 60\% de la matrícula, 70 instituciones se reparten el $40 \%$ restante, y de éstos, la mayoría reporta una matrícula total de 300 o menos estudiantes. 
de abandono en primer año se sitúa alrededor del 38\%, 12 puntos más alta que la existente en las carreras de tipo universitario. Con todo, de manera particular en los últimos años, se observa un mayor dinamismo en el comportamiento de la matrícula de este sector, reflejado en un aumento de más de 32.000 plazas entre el año 2005 y 2008, lo que significa un crecimiento cercano al 34\% durante este periodo. Es factible que este último hecho se explique por la reciente apertura a becas y créditos de carácter público para quienes cursan estudios en este sistema; de igual modo, como se verá en los antecedentes recogidos en el estudio, esta situación también puede explicarse, por lo menos en parte, por el incremento de la demanda hacia este tipo de carreras de estudiantes de mayor edad, muchos de ellos insertos por un tiempo prolongado en una actividad laboral, y que encuentran en este espacio una alternativa de certificación de estudios funcional a sus necesidades actuales, mayormente alineados a requerimientos del mercado laboral que exige mano de obra calificada y con conocimientos actualizados de sus respectivos sectores productivos.

Algunos análisis precedentes sobre el sistema de CFT han destacado los principales nudos críticos asociados a la oferta existente, entre otros, la gestión ineficiente de un segmento importante de centros vigentes, el escaso financiamiento público a este tipo de carreras, la débil vinculación de un número importante de centros formativos con el sector productivo, la carencia de mecanismos de articulación con el sistema universitario, y deficiencias curriculares que inciden en la superposición de contenidos con la enseñanza media técnicoprofesional (Bernasconi, 2006; Álamos, 2002). Esta situación se ve reforzada por las débiles señales observadas en el sistema productivo en la perspectiva de generar una demanda por profesionales de este sector formativo y el desconocimiento existente en el grueso de los empleadores acerca de las competencias ocupacionales efectivas que pueden ofrecer los programas formativos de nivel técnico (Araneda, 2006).

Con todo, algunas lecturas recientes destacan la importancia de un análisis desagregado de las ofertas formativas existentes las que, comparativamente, constituirían alternativas atractivas de inserción 
laboral y nivel de ingresos, incluso por encima de un segmento de carreras universitarias. Si a esto se agrega el menor costo promedio de estudios en este tipo de carreras técnicas y la estructura formativa curricular más cercana a los intereses y necesidades inmediatas del mundo productivo, el juicio evaluativo predominante sobre los CFT debería ser reconsiderado por lo menos en parte, evitando los prejuicios que han caracterizado el debate en los últimos años (Meller, 2010).

En este contexto, el reconocimiento del perfil y trayectoria de los estudiantes, así como sus intereses y motivaciones personales para ingresar a una carrera de nivel técnico, cuestión hasta ahora débilmente considerada, permitiría complementar el marco de antecedentes para una discusión informada sobre este campo formativo. Aunque algunos estudios recientes han ampliado la perspectiva analítica abordando este tema a partir de información secundaria (Paredes y Hernández, 2007; Sánchez y Escudero, 2008), en general no existen antecedentes empíricos precisos sobre las principales características de los estudiantes, y algunos estudios que abordan marginalmente este tema, entregan versiones contradictorias sobre información básica referida al origen y composición de la matrícula de este sector.

En términos generales, en la literatura sobre educación en general, y educación superior en particular, existe una escasa consideración de las estrategias individuales de los sujetos (los estudiantes y su entorno familiar), las expectativas y motivos de elección de una opción formativa y los eventuales proyectos que sustentan esta opción. La consideración de esta perspectiva debería ampliar la mirada sobre las lógicas sociales que orientan la demanda educativa, superando una mirada restrictiva, centrada exclusivamente en el orden institucional que enmarca la oferta formativa.

En perspectivas de realizar un aporte en tal dirección, en este artículo se entregan los principales resultados de un estudio dirigido a caracterizar la demanda social por formación técnica hacia este sector ${ }^{9}$, esto es, las características particulares de los estudiantes que ingresan

9 Estudio "Origen, expectativas y proyecto educativo-laboral de jóvenes estudiantes de centros de formación técnica", realizado entre septiembre de 2009 y junio de 2010. 
a las diversas carreras ofrecidas por estos centros formativos, los motivos de su inserción en esta modalidad de estudio y sus intereses educativo/laborales de futuro. La demanda formativa es entendida aquí como una racionalidad social (decisión que implica el ajuste de expectativas en función a la oferta formativa existente) como parte de un plan personal de formación postsecundaria. Tal demanda debe diferenciarse de la demanda económica, que generalmente se utiliza para indicar las tendencias y señales del mercado laboral y que, eventualmente, pueden incidir en el quehacer de los actores.

El objetivo principal de la indagación de este estudio consistió en revisar las trayectorias educativas de quienes ingresan a carreras de CFT, analizando el nivel de consistencia y grado de alineamiento que existe entre este ámbito de formación técnica superior y la formación media técnico-profesional; de igual modo, el estudio tuvo por objetivo recoger las expectativas y proyectos de mediano y largo plazo de los estudiantes, a fin de analizar la pertinencia de la oferta existente respecto a la subjetividad, intereses y metas que estos estudiantes elaboran. Esta información intenta aportar elementos significativos sobre el nivel de construcción vocacional de los estudiantes de carreras técnicas ampliando, de este modo, la base de antecedentes para la orientación de políticas destinadas al fortalecimiento del sector.

\section{Perspectiva de análisis}

Los cambios ocurridos en el mercado laboral en las últimas décadas, el declive de la oferta de puestos de trabajo para jóvenes egresados de la enseñanza secundaria, el debilitamiento de la noción de carrera laboral y el requerimiento de una formación a lo largo de la vida han incidido, de manera relevante, en el aumento de la presión por el ingreso a la educación superior, generando un fenómeno de masificación y diversificación de la oferta de credenciales para una demanda crecientemente más compleja en origen social y edad (Dubet, 2005).

Este nuevo escenario de la educación superior ha llevado a relevar dos temas fundamentales en la discusión sobre sus desafíos presentes y futuros: (a) en el ámbito de la oferta, la necesidad de 
analizar qué deben aprender los estudiantes en un escenario socioeconómico como el actual, qué tipo de estrategias formativas son necesarias para dar respuesta a una realidad dinámica y cambiante y cuáles son los mecanismos de apoyo y/o regulación que deberían impulsarse ante el desarrollo de nuevos sistemas de educación masiva, diseñados para satisfacer las necesidades de las economías emergentes; y (b) junto a lo anterior, en el ámbito de la demanda, resulta de gran relevancia reconocer nuevas pautas de transición que dan cuenta de la experiencia educativa de las nuevas generaciones, caracterizada por un menor soporte de estructuras y mecanismos institucionales, la mayor predominancia del sujeto en la tarea de diseñar sus propios itinerarios de vida, la diversificación de modelos y alternativas de transición entre la educación y el mundo del trabajo, no siempre compatibles con el orden institucional del sistema de formación terciaria (Heinz, 2009).

El debate sobre la situación y perspectiva de la educación superior en Chile ha estado mayormente enfocado en el primer tema, esto es, la consideración sobre la oferta en función a las necesidades de desarrollo del país (Edwards, 2005; OCDE-Banco Mundial, 2009), pero con una menor consideración del ámbito de la demanda. En este campo, la literatura internacional advierte, justamente, acerca de la diversidad y complejidad de la experiencia y trayectoria de los nuevos estudiantes de educación superior y la necesidad de reconocimiento de su experiencia para una reflexión sobre eventuales políticas dirigidas al sector (Furlong y Cartmel, 2009).

La preocupación por las trayectorias de vida, los procesos de adecuación de expectativas a las condiciones y oportunidades del entorno, así como el análisis de la interacción entre condiciones estructurales y agencia en las experiencias biográficas, constituye un campo de investigación creciente en los últimos años y que se enmarca en un modelo amplio de estudios conocido como estudios de life course (Heinz y Kruger, 2001).

En términos generales, los estudios bajo esta perspectiva reconocen que los cambios globales acaecidos en los últimos decenios a nivel socioeconómico han modificado radicalmente la noción lineal 
de la transición de la adolescencia/juventud a la adultez, entendida como transición unívoca de una fase de preparación previa para ingresar al mundo del trabajo. A diferencia de este modelo de transición lineal, en la actualidad predomina una perspectiva analítica que reconoce la complejidad de trayectorias posibles en la experiencia de los estudiantes (lo que pone en cuestión, de hecho, la propia noción de juventud como un periodo de edad delimitado) y el peso significativo que alcanza la experiencia de biografización ${ }^{10}$ de estas trayectorias (Du Bois-Raymond y López, 2004).

Desde esta perspectiva analítica se resaltan ciertos rasgos predominantes de la actual experiencia de transición educación/ trabajo de los sujetos: (a) las transiciones de vida, el paso de una etapa vital a otra (por ejemplo de estudiante secundario a estudiante postsecundario), aunque enmarcada en condicionamientos estructurales, adquiere una dimensión mayormente individualizada, referida a las expectativas, estrategias y capital específico que acumula el sujeto por sobre las orientaciones colectivas de tipo transversal; (b) crecientemente se observa una pérdida de sentido de la transición como una secuencia normativa y lineal, pudiendo existir entradas y salidas de un sistema o búsqueda de alternativas intermedias que posteriormente implican cambios en la orientación de los sujetos; (c) muy asociado a esto, no existe un modelo único de transición o de construcción de carrera hacia el futuro, prevaleciendo una diversidad de experiencias sin que una se imponga sobre la otra a partir de una eventual mayor eficacia o éxito; y (d) la transición se alarga temporalmente posponiendo, para una gran masa de jóvenes, la asunción de roles tradicionales en lo que comúnmente se denomina la extensión de la moratoria, esto es, la indefinición del cierre o término de un proceso formativo (Heinz y Marshall, 2003; Staff y Mortimer, 2003).

Estos rasgos inciden, o deberían incidir, de manera considerable en la propia dinámica de la oferta educativa; estudiar una carrera de

10 Con este concepto, los autores dan cuenta del debilitamiento de los canales institucionales tradicionales de organización social, de tal forma que la mayoría de los sujetos tiene que construir su propia biografía personal, sin depender de los elementos de contexto o las tradiciones sociales precedentes para alcanzar un objetivo de vida adulta. 
nivel superior ya no puede ser considerado un evento temporalmente situado en la experiencia biográfica de las personas, como de la misma manera, la realización de estudios o la finalización de una carrera no constituye, necesariamente, una meta u objetivo terminal en la trayectoria individual. Pese al creciente reconocimiento de este hecho, el sistema institucional de la oferta y las políticas nacionales de fortalecimiento formativo presentan importantes rezagos para adoptar un modelo formativo funcional a las nuevas pautas de vida y nuevos requerimientos de quienes hoy ingresan al sistema de educación superior (Wyn y Dwyer, 2000).

El reconocimiento de las pautas de transición predominantes en los actuales estudiantes así como la distinción de trayectorias vinculadas a factores de origen social, género o edad representa, de este modo, un tipo de información que debería ser considerada, con mayor insistencia, en los diseños y la discusión pública de la oferta formativa de nivel superior y de la educación técnica en particular, con el objetivo de consolidar un sistema funcional a las transformaciones verificadas en el entorno social. Este foco de interés es el que guía la indagación cuyos principales resultados aquí se presentan.

\section{Antecedentes del estudio}

El estudio consistió, básicamente, en la aplicación de un instrumento estructurado a una muestra representativa de estudiantes que cursaban el primer año de una carrera de nivel técnico superior en CFT de la Región Metropolitana durante el año 2009.

La construcción de la muestra se definió en dos etapas. En la primera se sorteó un número fijo de instituciones-carreras (u ofertas formativas) que actualmente se dictan en los diversos CFT de la Región Metropolitana (de acuerdo con la base de datos del Consejo Nacional de Educación, en el año 2009 existían 401 ofertas formativas en el sistema). La segunda etapa consistió en la selección de un grupo curso de cada una de las carreras seleccionadas y, dentro de éste, se definió una cuota de un máximo de 15 estudiantes cursando el primer año de la respectiva carrera para ser encuestado (este número es estimado, ya que (a) no existen bases de datos para definir con 
mayor precisión el conjunto de los encuestados en este caso, y (b) la población estudiantil en las distintas carreras sufre modificaciones a lo largo del tiempo debido a la dinámica de deserción).

Como producto de un trabajo de terreno que se extendió entre los meses de octubre de 2009 y mayo de 2010, la indagación permitió aplicar 901 encuestas a estudiantes que cursaban 95 carreras de los distintos sectores de especialidad formativa correspondientes a 23 CFT en funcionamiento.

La encuesta, de carácter autoaplicada, incluyó un conjunto de indicadores orientados a la caracterización sociocultural de los estudiantes, sus trayectorias educativo-laborales previas al ingreso al CFT, la experiencia de trabajo y situación laboral actual, los motivos de elección de la carrera y CFT donde estudian, así como la evaluación de la formación recibida y las perspectivas de futuro personal en el corto y mediano plazo.

\section{Principales resultados}

Los resultados de este estudio permiten advertir acerca de la existencia de rasgos socioculturales específicos de los actuales estudiantes de las carreras de CFT, destacando (a) la heterogeneidad en la composición etaria de la matrícula y la, comparativamente, baja incidencia de población estudiantil que ingresa a una carrera inmediatamente después de haber finalizado sus estudios medios; (b) el débil vínculo que se observa entre la formación técnica de la enseñanza secundaria respecto a la oferta de nivel superior; (c) la diversidad de modelos de trayectorias observables en la experiencia de los actuales estudiantes, y el peso significativo que tiene la experiencia laboral previa de un segmento importante de éstos; (d) la situación actual de combinación de estudios con una actividad laboral para un grupo relevante de los estudiantes; (e) las distinciones en los motivos de elección de una carrera CFT y las condiciones de financiamiento de los estudios técnicos, y (f) el proyecto de futuro y la preeminencia de la extensión de los estudios en el proyecto personal de la mayoría de los estudiantes, independientemente de la edad y situación laboral de éstos. 
(a) Composición de la matrícula: heterogeneidad etaria y sesgo de género

Uno de los aspectos más relevantes entre los antecedentes recogidos en este estudio, tiene que ver con el perfil etario de los estudiantes de primer año de las carreras que imparten los distintos CFT. De acuerdo con los antecedentes recogidos, menos de un tercio de quienes cursan alguna carrera de este nivel tiene una edad igual o inferior a los 20 años, mientras que casi el $40 \%$ de los estudiantes de este nivel ingresa a la carrera con una edad igual o superior a los 25 años. Lo anterior indica que el grueso de los estudiantes del sistema no ha realizado una transición inmediata de la educación media a la educación técnica de nivel superior, existiendo un historial de trayectoria más complejo para la gran mayoría de quienes se encuentran en el primer año de una carrera de formación técnica.

Gráfico n ${ }^{1}$. Distribución matrícula estudiantes CFT por edad.

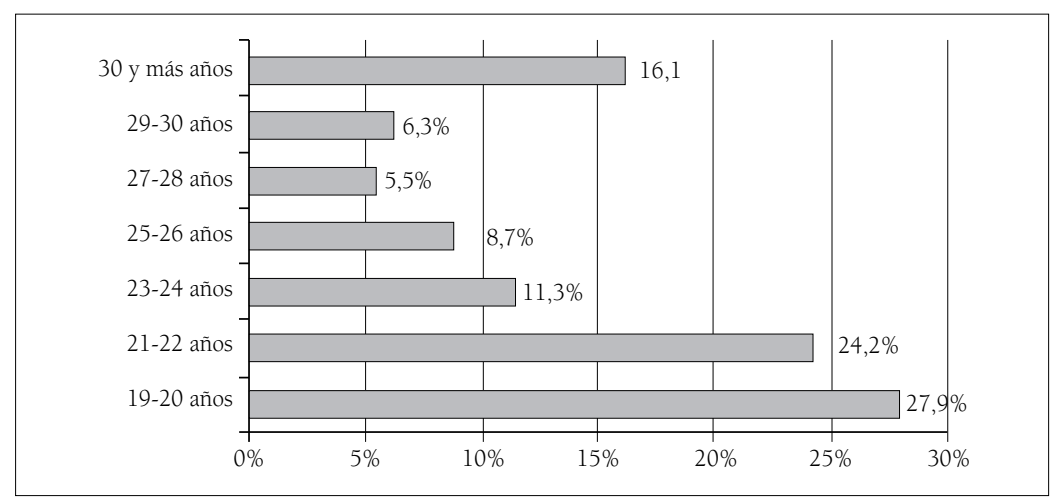

Aunque los datos contrastan con algunos antecedentes previos sobre el perfil de la matrícula de CFT, que indicaban una mayor preponderancia de situaciones de transición lineal desde la enseñanza media a carreras de este modelo formativo (Donoso y Cancino, 2007), este hecho puede ser un indicador de una tendencia reciente de presión hacia el sistema de educación superior en el país. El informe de la OCDE sobre Educación Superior en Chile advierte acerca de esta situación; mientras que en el año 1998 el 22,6\% de los estudiantes de educación superior tenía 25 años o más, en el año 2003 la participación de la población adulta había crecido a un 30,3\%, 
especialmente en el sector no universitario (OCDE, Banco Mundial, 2009). Es factible pensar, desde este punto de vista, que en la última década la expansión de la matrícula de la educación superior ha estado asociada, entre otros factores, al incremento de estudiantes que han desarrollado una trayectoria irregular ${ }^{11}$ de combinación de estudios y/o trabajo; el caso de los CFT puede ser un ejemplo relevante en esta dirección acercándose, por lo menos en esta dimensión, a lo observado en el modelo de institutos técnicos superiores norteamericano (De Moura y García, 2003) ${ }^{12}$.

Por otra parte, a partir de los resultados del estudio también es posible observar una correspondencia entre la categoría de edad y la modalidad de horario de estudios que ofrece el sistema; así, casi el 80\% de los estudiantes de hasta 20 años estudia bajo la modalidad diurna, porcentaje que se reduce al 69,8\% de los estudiantes de 21 a 25 años. Las mayores diferencias a favor de cursar una carrera en la modalidad vespertina, como es previsible, se encuentra en las categorías de 26 años en adelante, donde el 55,2\% de los estudiantes entre 26 y 30 años y el 64,3\% de quienes tienen más de 30 años lo hacen en esta última modalidad debido, fundamentalmente, a la realización de alguna actividad laboral remunerada. Con todo, la realización de estudios bajo la modalidad vespertina no es un patrimonio exclusivo de los estudiantes de mayor edad; como podrá verse más adelante, la combinación de estudios y trabajo es una característica que está presente también en un porcentaje importante de los estudiantes más jóvenes, incluyendo aquellos que realizan sus estudios bajo modalidad diurna.

11 Llamamos trayectoria irregular al conjunto de modelos existentes de transición que combinan, en un periodo indefinido de la vida de los sujetos, actividades de formación y experiencia laboral. La noción de trayectoria irregular o compleja constituye una herramienta conceptual de base empírica que intenta ampliar la noción tradicional de transición lineal secundaria-educación superior o secundaria-trabajo predominante bajo otro modelo de desarrollo socioproductivo y, sin embargo, aún fuertemente presente en la organización de modelos formativos de nuestra enseñanza media, como se refleja en la distinción de las modalidades de educación media científico-humanista y técnico-profesional.

12 Hacia fines de la década de los 90, el modelo norteamericano de institutos técnicos superiores presentaba un perfil de matrícula de un promedio de edad de 29 años; el 64\% de los estudiantes asistía a cursos de tiempo parcial y el $84 \%$ trabajaba en empleos remunerados de jornada completa. Por lo mismo, una parte importante de la oferta formativa se dicta en horario nocturno o en fines de semana y existe una organización física de sedes de los centros formativos asociados a lugares de residencia de los estudiantes. 
La composición de la matrícula de los CFT es relativamente proporcional entre hombres y mujeres, sin embargo, al cruzar sexo y edad, se observa una mayor proporcionalidad de mujeres entre el grupo de estudiantes más jóvenes (entre 19 y 24 años), cuestión que se revierte a partir del grupo de 25 o más años de edad. Como puede verse en el gráfico adjunto, casi el $70 \%$ de las mujeres tiene 24 o menos años, 14 puntos más que la población masculina en ese tramo de edad. En el grupo de 30 años o más existe una diferencia de casi 7 puntos a favor de la población masculina.

Gráfico n. ${ }^{\circ}$ 2. Distribución matrícula estudiantes CFT por sexo y edad.

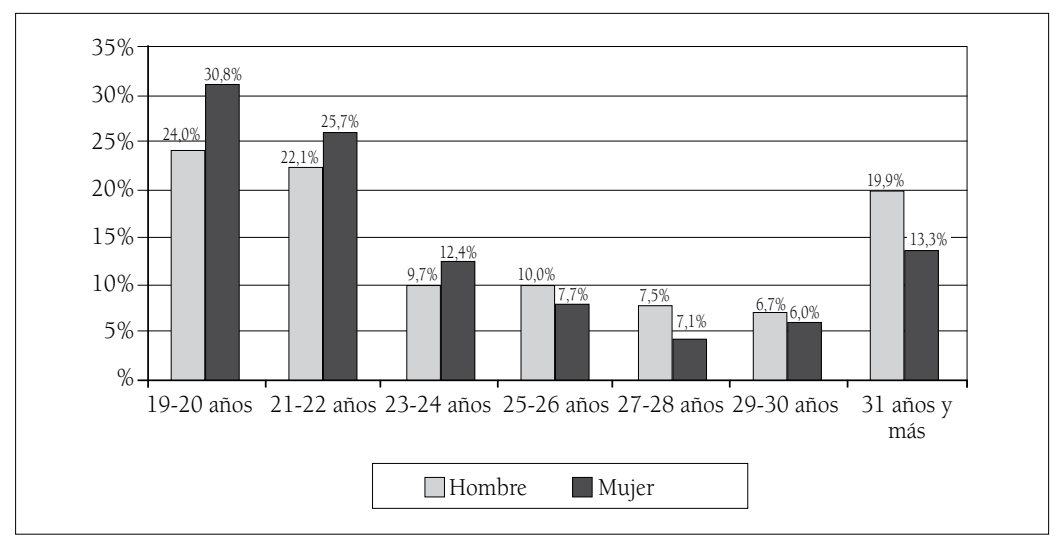

Es importante anotar que este sistema de formación es el que presenta la mayor brecha de retención considerando la variable género; mientras que el conjunto de las instituciones de educación superior reporta una diferencia en la tasa de retención de 5,1, en el caso de los CFT, la diferencia se eleva a $6,6^{13}$.

Tal situación se vincula con las diferencias observadas en la composición de la matrícula de las carreras de carácter diurno respecto a las vespertinas; para el caso de los CFT, la tasa de retención de las carreras diurnas alcanza al $68,4 \%$ con una diferencia de 7,7 puntos entre hombres y mujeres (tasas de $64 \%$ y $71,1 \%$, respectivamente); en el caso de las carreras vespertinas la brecha es de 5,2 entre hombres y mujeres ( $52 \%$ y $57,5 \%$, respectivamente). Esta información es relevante 
ya que evidencia no sólo la brecha existente desde un punto de vista de género, sino también la especificidad de las modalidades formativas.

Así, estas diferencias definen un sello particular al actual sistema de formación de los CFT, evidenciándose una distinción que obliga a hablar de dos modelos tipo de estudiantes de educación técnica de nivel superior: estudiantes más jóvenes y mayoritariamente mujeres en la modalidad diurna, y estudiantes de mayor edad y mayoritariamente hombres bajo la modalidad vespertina, quienes presentan, además, una mayor predisposición al abandono de sus carreras antes de su finalización.

Finalmente, los antecedentes recogidos en el estudio señalan que un poco más del $75 \%$ de los estudiantes es soltero(a); el 26,5\% tiene a lo menos un hijo, y más de dos tercios de quienes cursa una carrera aún vive en el hogar de su familia de origen (79,8\% del total de la muestra) con una mayor predominancia de las mujeres $(75,1 \%)$ respecto de los hombres (64,8\% del total de la población masculina).

Un aspecto relevante de la información obtenida es que aunque existe una mayor incidencia de la conformación de un hogar autónomo en la población estudiantil de mayor edad, en sentido estricto, ésta sólo es una situación mayoritaria en el caso de los estudiantes que superan los 30 años de edad, evidenciando un rasgo distintivo de la condición juvenil actual, esto es, la extensión de un periodo de semiautonomía y permanencia en el hogar de origen por un tiempo prolongado.

Tabla n. $^{\circ} 1$. Distribución estudiantes por tipo de hogar donde vive por tramo de edad.

\begin{tabular}{|c|c|c|c|c|c|}
\hline \multirow{2}{*}{ Tipo de hogar } & \multicolumn{5}{|c|}{ Edad en tramos } \\
\cline { 2 - 6 } & Hasta 20 años & 21 a 25 años & 26 a 30 años & 31 años y más & Total \\
\hline Hogar con familia de origen & $91,5 \%$ & $78,9 \%$ & $55,1 \%$ & $29,4 \%$ & $70,8 \%$ \\
\hline Hogar autónomo & $8,5 \%$ & $21,1 \%$ & $44,9 \%$ & $70,6 \%$ & $29,2 \%$ \\
\hline
\end{tabular}

La gran mayoría de estos estudiantes, además, al cursar su primer año de estudios técnico-profesionales ha superado el nivel educativo de sus padres; según los antecedentes recogidos, el 74,2\% de los padres y el 77,7\% de las madres de los encuestados alcanzaron, como máximo, los 12 años de escolaridad, y de éstos, el 21,8\% de los padres y $25,3 \%$ de las madres no alcanzaron estudios de nivel medio. 
Esta última información permite contar con una aproximación para caracterizar la situación socioeconómica de los estudiantes del sistema (sobre la base de la relación existente entre nivel educacional del grupo familiar y situación socioeconómica); tal como lo indica el Informe de la Comisión Asesora Ministerial para la Formación Técnico-Profesional, los CFT cuentan, comparativamente, con una mayor proporción de estudiantes provenientes de los estratos de menores recursos; el 51\% de los estudiantes de CFT pertenece a los tres primeros quintiles de ingresos, porcentaje similar a los estudiantes pertenecientes a IP, y 13 puntos más pobres que los estudiantes de carreras universitarias (Comisión asesora de formación técnico-profesional, 2009).

(b) Estudios previos: debilidad de puentes o itinerarios formativos en la enseñanza técnico-profesional

Un segundo aspecto destacable de los resultados alcanzados en el estudio tiene que ver con la débil incidencia de la educación técnica de nivel medio en la matrícula de esta modalidad de enseñanza de nivel superior. Los antecedentes sobre trayectoria educativa de los estudiantes de CFT indican que el 54,8\% del total de quienes ingresan al sistema estudió bajo la modalidad de enseñanza media científico-humanista, mientras que sólo el 45,2\% de éstos lo hizo bajo la modalidad técnicoprofesional, por lo que no es evidente la existencia de una línea de continuidad o puente entre los estudios técnicos de nivel secundario y la elección de una carrera en el nivel de formación técnica superior. Desde este punto de vista, los antecedentes de estudios previos de nivel secundario no constituyen un factor de incidencia relevante para la realización de estudios en una carrera impartida por un CFT.

Los antecedentes proporcionados sobre trayectoria educativa previa señalan que el 76,2\% de los actuales estudiantes de CFT habría tenido un egreso oportuno de la enseñanza media (no habría repetido ninguno de los niveles de la enseñanza básica y media), aunque existen diferencias importantes al considerar la categoría de edad ${ }^{14}$. Respecto

14 Mientras que sólo el 9\% los estudiantes de 20 años o menos señala haber repetido en algún nivel de su enseñanza obligatoria, este porcentaje se incrementa sucesivamente en los tramos de edad siguientes; crece al 26,5\% en el grupo de 21 a 25 años, $32,8 \%$ en el grupo de 26 a 30 años y $34,3 \%$ en el caso de quienes señalan tener 31 o más años. 
a la situación específica del segmento de estudiantes que cursaron su enseñanza media en la modalidad de educación técnico-profesional, puede observarse que existe una reducción importante del número total de estudiantes que finaliza el proceso conducente a la obtención de su título profesional.

En concordancia con la información disponible sobre este tema en otros estudios ${ }^{15}$, cuatro de cada diez egresados bajo esta modalidad, no finaliza el proceso de titulación que está asociado a la realización de una práctica profesional en un periodo posterior a la finalización de su proceso de formación obligatoria de la enseñanza media. Este hecho, por cierto, puede explicarse por varias razones, incluyendo el desajuste vocacional de un segmento de estudiantes egresados de la EMTP, la postergación del inicio de la vida laboral en el proyecto inmediato de los egresados del sistema, como también, la baja valoración de la credencial de los técnicos de nivel medio en el país, cuestión que puede desincentivar el cumplimiento de esta instancia luego del egreso de cuarto año de la enseñanza media.

Los datos recogidos en este estudio señalan que sólo un 43,4\% del total de quienes provienen de la enseñanza técnico-profesional eligió una carrera que se encuentra dentro del mismo sector de especialidad de la formación recibida en la enseñanza media ${ }^{16}$, ratificando lo ya señalado acerca de la débil vinculación entre la experiencia formativa de nivel técnico en la enseñanza secundaria respecto a la opción de estudios observada en este sistema de educación superior. Esta situación presenta algunas diferencias al analizar el grupo de estudiantes de acuerdo con la variable edad. El grado de coherencia de los estudios es mayor entre quienes han finalizado recientemente su formación de nivel secundario; un poco más del $67 \%$ de quienes se encuentran en esta situación estudia carreras en concordancia con su formación previa a nivel EMTP. A diferencia de lo anterior, quienes tienen 25 o más años presentan una mayor brecha en este ámbito temático, donde sólo el 30\% de quienes

15 Véase Sepúlveda, et al. (2009).

16 La coherencia de estudios se realiza comparando la especialidad EMTP estudiada y la clasificación de la respectiva carrera de CFT a partir de las categorías de especialidades definidas por MINEDUC. 
se sitúan en este rango de edad presenta una situación de coherencia en sus estudios medios y postsecundarios.

Más allá de lo anterior, la información recogida reafirma el débil vínculo entre la formación técnica de nivel medio con el área o temática de la opción de estudios en los respectivos CFT. De este modo, se refuerza la impresión de que el sistema de educación técnica superior que se imparte en estos centros formativos, menos que representar una oferta vocacional en línea o una ruta lógica para los estudiantes de enseñanza media que estudian carreras de formación técnicoprofesional, constituye una modalidad de formación alternativa que opera, en un porcentaje alto de casos, como la apertura a un nuevo campo de interés o posibilidades, así como también, una alternativa de segunda (o tercera) oportunidad para quienes tienen un periodo prolongado de egreso de la enseñanza media sin contar con ningún tipo de certificación de estudios postsecundarios.

\section{(c) Experiencia previa: trayectorias disímiles y predomino de actividad laboral}

La gran mayoría de los estudiantes que cursan el primer año de una carrera de CFT ha tenido una experiencia educativa y/o laboral previa a esta situación. Del total de los estudiantes encuestados para este estudio, sólo el 21\% ingresó directamente a su carrera luego de haber finalizado sus estudios de enseñanza media; el resto, experimentó diversas trayectorias individuales de estudio y trabajo (como también modelos de trayectorias que combinan ambas a la vez), constituyendo el ingreso a la carrera técnica una etapa posterior, aunque no necesariamente terminal, de este proceso ${ }^{17}$.

Pese a que esta situación de no linealidad, debido a su magnitud, se expresa como una realidad transversal para el conjunto de los estudiantes del sistema, de todos modos es posible observar algunas diferencias en la distribución de éstos considerando las variables más relevantes. Así, mientras que el 23,3\% de las estudiantes mujeres

17 La consideración de ingreso directo a la carrera de CFT corresponde a quienes en diciembre del año anterior se encontraban cursando el último año de su enseñanza media. De este modo, para el $79 \%$ de la muestra había transcurrido, a lo menos, un año completo de egreso de la enseñanza media antes de ingresar a su actual carrera en el respectivo CFT. 
ingresa inmediatamente después de haber finalizado su enseñanza media a una carrera de CFT, en el caso de los hombres, este porcentaje se reduce al $16,3 \%$, ratificando lo ya señalado de una mayor proporción de mujeres jóvenes en la composición de la matrícula del sistema.

En el caso de la distribución de estudiantes por sector de especialidad estudiado ${ }^{18}$, también es posible identificar algunos matices. De este modo, sólo un 15\% de los estudiantes que cursan carreras del sector de administración y comercio ingresó directamente después de haber finalizado su enseñanza media, porcentaje similar al observado en el caso de los estudiantes de carreras del sector de tecnología (18,7\% del total). A diferencia de esto, existe una mayor proporción de casos de tránsito directo entre los estudiantes de carreras del sector salud, porcentaje que alcanza al 32,3\% del total. En efecto, las carreras del sector salud concentran un $36 \%$ de su matrícula con estudiantes de menos de 20 años, esto es, casi 10 puntos más que las carreras de los otros sectores de especialidad con mayor matrícula del sistema. Aunque resulta arriesgado sostenerlo de manera taxativa considerando sólo estos datos, los antecedentes permitirían hipotetizar que, en términos comparativos, las actuales carreras del sector salud pueden resultar más atractivas para los jóvenes egresados de la enseñanza media y que ingresan de inmediato a carreras de la educación técnica superior.

De igual manera, cuando se hace la distinción según el carácter de las instituciones donde estudian los encuestados (CFT por régimen de licenciamiento), también se observan diferencias importantes. En este caso, los estudiantes en situación de tránsito directo (ingreso a la carrera inmediatamente después del egreso de la enseñanza media) de los CFT autónomos casi duplican a aquellos que cursan sus estudios en centros no autónomos ${ }^{19}$. Lo anterior podría indicar una

18 Las carreras impartidas por los CFT concentran su matrícula, básicamente, en tres sectores o áreas de conocimiento: administración y comercio, tecnología y salud, representando el $81 \%$ del total de los estudiantes del sistema.

19 Las instituciones de educación superior de carácter autónomo son aquellas que están facultadas para otorgar los títulos y grados que corresponden a la carrera impartida de manera independiente; las instituciones en licenciamiento (o en acreditación) son aquellas que aspiran a obtener su autonomía, que es otorgada por el Consejo Nacional de Educación. Por otra parte, también existen centros de formación técnica creados antes de 1990 y que no han optado por el licenciamiento; éstos son denominados en supervisión, y tal tarea es desarrollada por el Ministerio de Educación. El MINEDUC debe velar por el cumplimiento de las condiciones mínimas de funcionamiento, y los CFT, bajo esta figura, no pueden aspirar a su autonomía. 
segmentación de mercado institucional, que favorece a los centros de mayor legitimidad institucional (en el sentido que la condición de autonomía así lo señala), acogiendo a los estudiantes más jóvenes y que realizan un tránsito directo desde la enseñanza media hacia esta modalidad de estudios. Por cierto, esta situación no puede aislarse de otros factores relevantes, como el costo de la carrera y el acceso a financiamiento de quienes estudian bajo este sistema.

Más allá de estas consideraciones, los antecedentes sobre la trayectoria previa al ingreso a las respectivas carreras ${ }^{20}$ señalan la fragmentación de los periodos estudiantiles para un número relevante de estudiantes de CFT. En efecto, un segmento importante de los encuestados, casi el 40\% de los estudiantes, sólo se dedicó a trabajar en los tres años previos al ingreso de su actual carrera. Un poco más de un quinto de los encuestados combinó actividades de estudios, trabajo o inactividad en ese periodo ${ }^{21}$, y sólo un 12\% de éstos realizó estudios de educación superior como actividad exclusiva. El grupo lo cierra un $5 \%$ de los estudiantes que en el periodo previo se mantuvo inactivo, sin desarrollar actividades de estudio ni trabajo ${ }^{22}$; el gráfico siguiente resume esta situación ${ }^{23}$.

20 Para analizar las trayectorias de quienes no entraron al CFT inmediatamente después de haber terminado su educación media (es decir, la gran mayoría de los estudiantes), se pidió a los encuestados que completaran una tabla de doble entrada en que se señalaba, para cada semestre anterior al ingreso al CFT, la actividad que habían realizado, lo que implicaba reconstruir su situación educativo/laboral de hasta tres años hacia atrás (inicios de 2006). De los 717 entrevistados que cuentan con una trayectoria previa al CFT, 612 entregaron información sobre las actividades realizadas en este periodo, lo que corresponde a un $85,4 \%$ del total de encuestados.

21 La combinación de estudios y trabajo incluye una amplia variedad de experiencias de entradas y salidas de situaciones parciales de trabajo y estudio a lo largo del periodo consultado.

22 Es necesario recalcar que la encuesta aplicada en este estudio sólo recogió la situación de los tres años previos al ingreso a la carrera de CFT de los respectivos estudiantes. Es sabido que la situación de inactividad tiene una incidencia importante en el primer año de egreso postsecundario; pero, debido a la particularidad del presente estudio, tal situación no se evidencia en su verdadera magnitud.

23 En el gráfico existe una diferencia de dos puntos en la referencia del porcentaje de casos que señala haber realizado una transición directa de la enseñanza media a la carrera CFT en comparación con los datos entregados anteriormente; esta situación se explica por la particularidad de la muestra sobre la que se construye esta información (véase nota n.․ 17). 
Gráfico n. 3 3. Distribución de estudiantes CFT por tipo de actividad previa al ingreso de actual carrera.

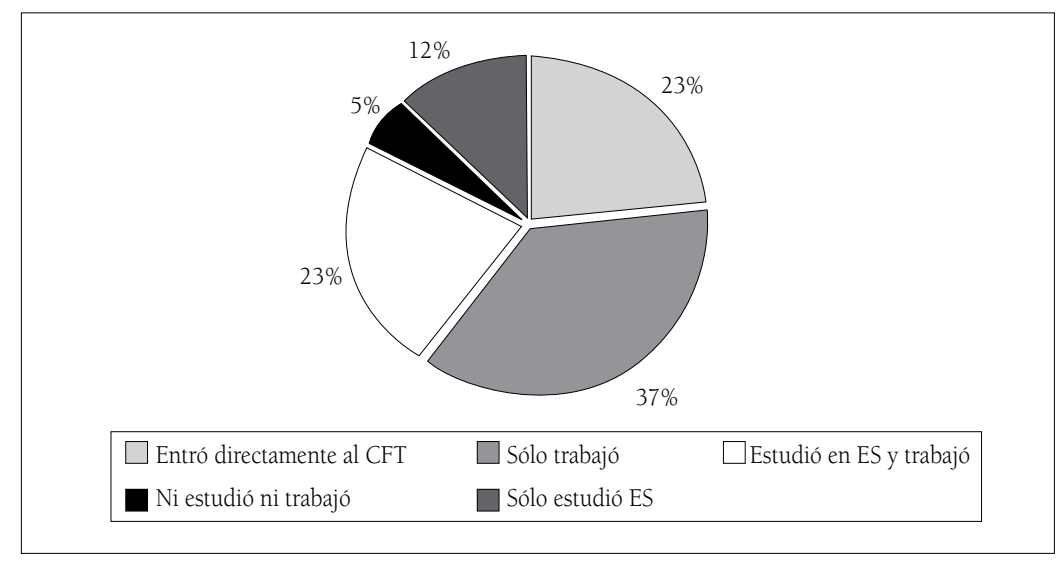

Revisar esta situación respecto a la categoría de edad de los estudiantes demuestra que la experiencia laboral prolongada, previa al ingreso a sus respectivas carreras, es una situación predominante entre los estudiantes de 25 años o más. Como puede observarse en el gráfico siguiente, salvo en el caso de la población menor de 20 años, que concentra la situación de tránsito lineal enseñanza mediaenseñanza superior, la experiencia de trabajo en la trayectoria de vida de la población estudiantil presenta una fuerte incidencia, muy por encima de quienes sólo han pasado por experiencias formativas; cerca de un tercio de los estudiantes de las categorías de edad por sobre los 21 años señala haber combinado estudios y trabajo en los años previos a su ingreso a su actual carrera técnica, por lo que resultan relativamente marginales los casos de dedicación exclusiva a los estudios de aquellos que presentan una experiencia previa al ingreso de sus respectivas carreras. 
Gráfico n. ${ }^{\circ} 4$. Distribución de estudiantes CFT por tipo de actividad previa al ingreso de actual carrera por categoría de edad.

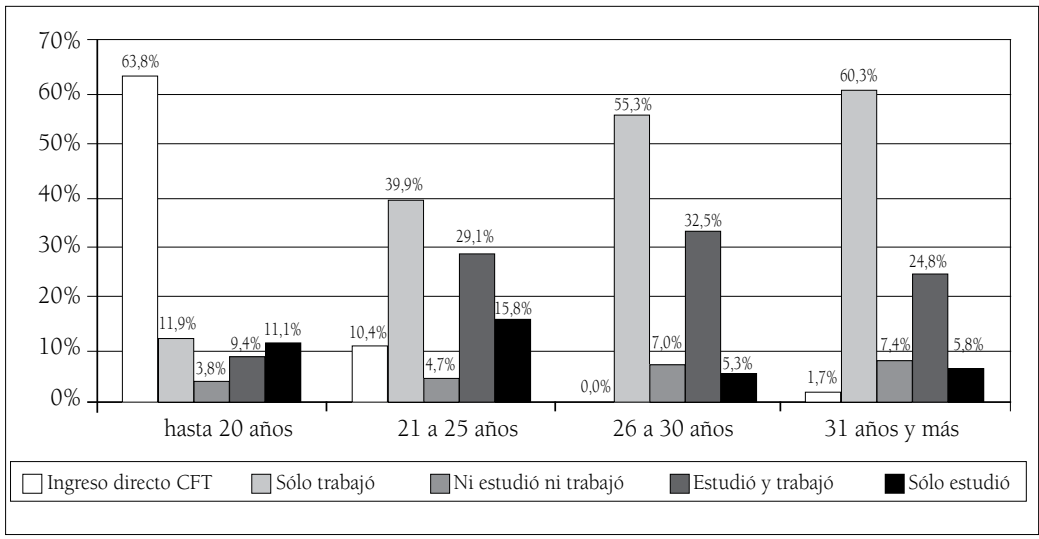

En función a los antecedentes expuestos, puede decirse que las trayectorias educativo/laborales de los estudiantes de CFT presentan variaciones importantes, fuertemente asociadas a la heterogeneidad etaria relevada en el estudio. Para un número significativo de estudiantes, el ingreso a su carrera no está precedido de una experiencia formativa previa, existiendo un espacio prolongado de tiempo destinado exclusivamente a la realización de una actividad laboral, siendo esta característica aun más marcada para quienes cursaron su enseñanza media en la educación técnico-profesional. Estos antecedentes, sumados a la baja recurrencia de casos que reflejan una continuidad de estudios técnicos medios con la actual experiencia estudiantil en los CFT, advierten sobre el carácter fragmentado de la experiencia de la mayoría de los estudiantes. El siguiente esquema sintetiza los modelos de trayectoria aquí revisados. 
84 TRAYECTORIAS DISÍMILES Y PROYECTOS INDIVIDUALIZADOS: ORIGEN Y EXPERIENCIA EDUCATIVO-LABORAL DE LOS ESTUDIANTES DE CENTROS DE FORMACIÓN TÉCNICA Leandro Sepúlveda, Pamela Ugalde

Esquema n. ${ }^{\circ}$ 1. Síntesis trayectorias educativa/laborales estudiantes CFT.

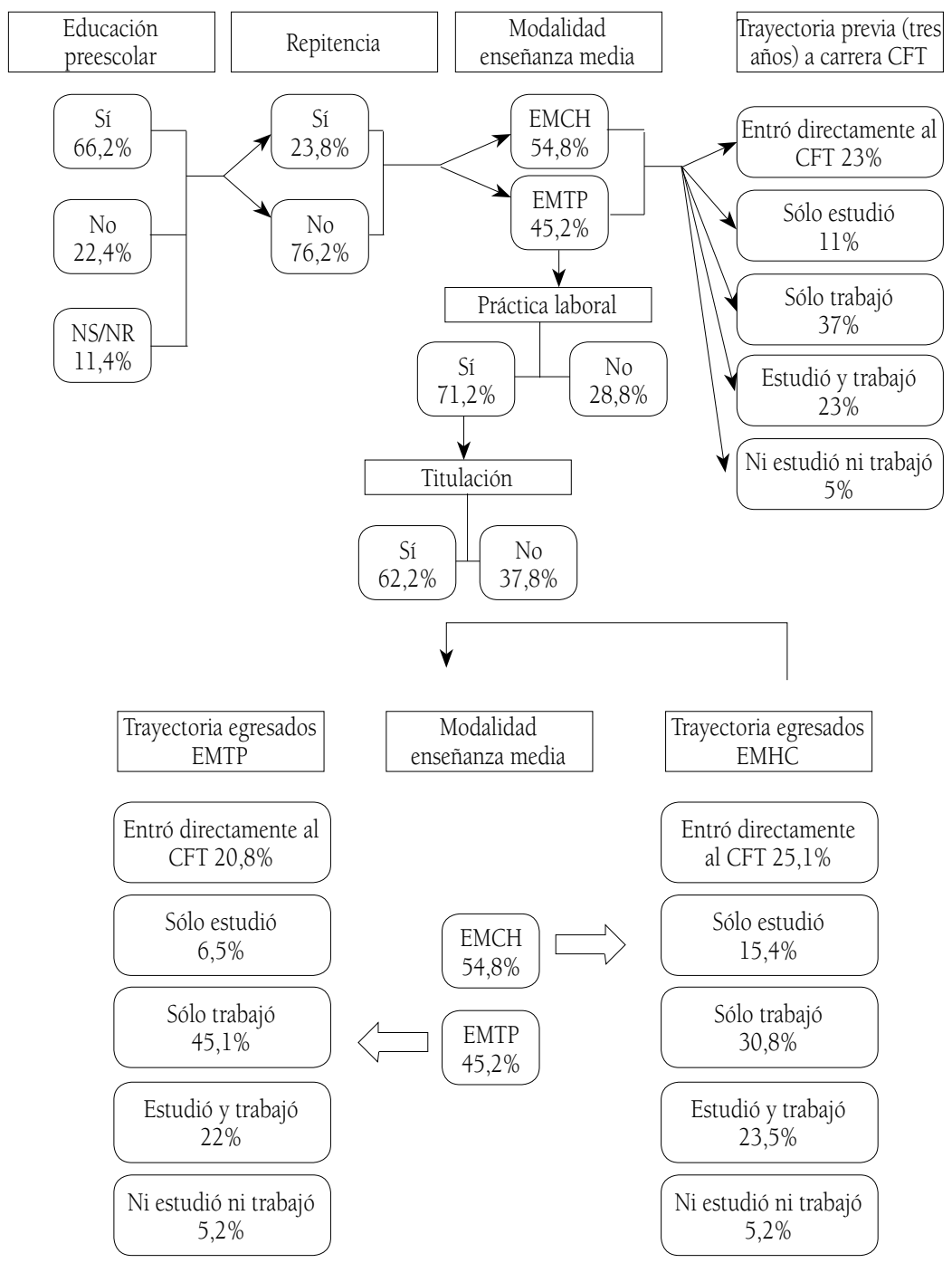

\section{(d) Experiencia laboral: combinación de estudio y trabajo en la actual condición estudiantil}

Los resultados de este estudio señalan que en la actualidad un 48,1\% de los estudiantes de las diversas carreras de CFT se encuentra actualmente trabajando. La proporción es mucho mayor entre 
hombres que entre mujeres; el 61,4\% de los hombres se encuentra en esta situación, mientras que en las mujeres, lo hace el 38,3\% de la muestra.

Al revisarse la situación laboral por categoría de edad, como es previsible, esta situación es mucho más recurrente entre los estudiantes por sobre los 25 años. Con todo, no es menor el porcentaje de casos que informa realizar alguna actividad laboral entre los estudiantes más jóvenes: un tercio de los estudiantes de menos de 20 años combina sus estudios con trabajo, y casi el 44\% de los jóvenes entre 21 y 25 años se encuentra en esta situación:

Gráfico n. ${ }^{\circ}$ 5. Distribución estudiantes CFT por situación laboral.

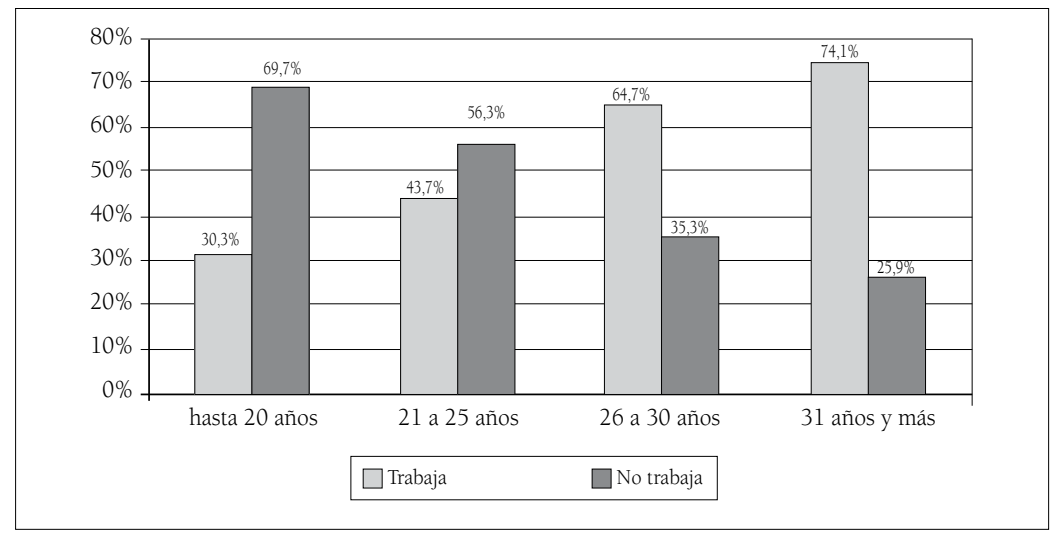

Al responder la interrogante sobre las horas semanales trabajadas, la mayoría de los estudiantes menores de 20 años señala que su actividad laboral semanal no excede las 30 horas; sin embargo en el grupo de edad de 21 a 25 años un poco más de la mitad informa desarrollar actividades laborales que le demandan más de 31 horas a la semana, cuestión que se incrementa en los grupos de mayor edad.

Como consecuencia, a partir de estos datos es posible sostener que el grueso de los estudiantes de CFT (incluyendo un segmento importante de quienes cursan su formación bajo la modalidad diurna) no sólo desarrolla una actividad laboral, sino también que ésta se desenvuelve en una jornada extensa, implicando el desafío de articular educación y trabajo en la organización de su vida cotidiana. El detalle 
de la información de los estudiantes que realizan alguna actividad laboral en combinación con sus estudios, por categoría de edad, se entrega en el siguiente gráfico:

Gráfico n. ${ }^{\circ}$ 6. Distribución de estudiantes de CFT que realizan actividad laboral por categoría de edad y horas de trabajo semanales.

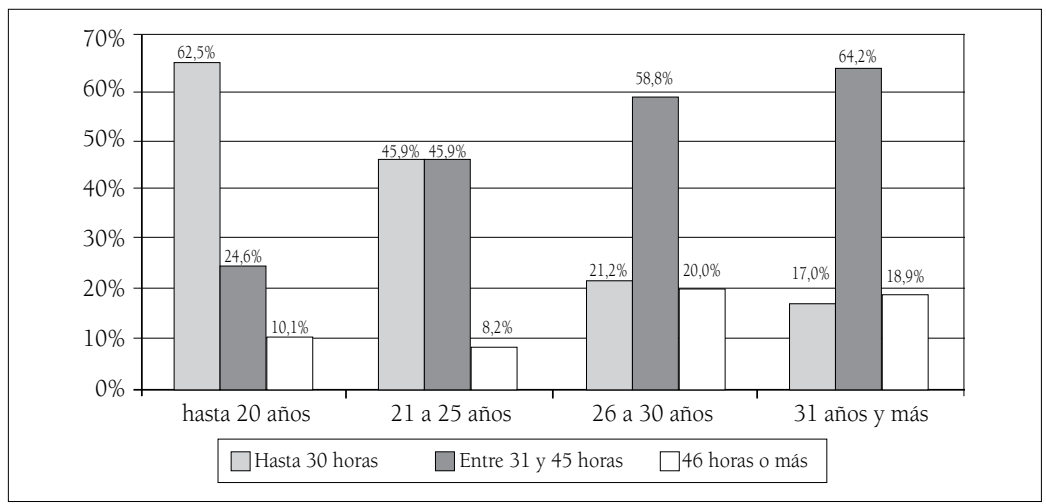

Finalmente, al revisar la situación laboral actual de los estudiantes (es decir, la combinación de estudios y trabajo o sólo la realización de estudios) en relación con la experiencia previa al ingreso al CFT, puede observarse una tendencia de continuidad en la trayectoria de los sujetos; en efecto, el 64,5\% de los estudiantes que proviene de una experiencia laboral previa (quienes declararon solo trabajar en el periodo previo al ingreso a su carrera) mantiene este estado en la actualidad; esto ocurre también con el 59\% de quienes declaran haber combinado estudios y trabajo antes de iniciar estudios en su actual carrera técnica.

En sentido contrario, aquellos estudiantes que no tienen una actividad laboral previa en su gran mayoría mantiene este estado; con todo, un $27 \%$ de quienes ingresaron a la educación técnica superior inmediatamente después de finalizados sus estudios medios se encuentra trabajando en la actualidad; un porcentaje similar se observa entre quienes sólo estudiaron en algún nivel de educación superior.

De este modo, la experiencia laboral es una situación extendida para un amplio porcentaje de estudiantes de carreras técnicas en la 
actualidad. La presencia significativa de estudiantes mayores con un trabajo relativamente estable permite señalar la presencia consistente de un modelo en que la elección de la carrera técnica se realiza desde la experiencia del trabajo y no al revés.

(e) Elección de la carrera y CFT, condiciones de estudio y proyección de futuro: la extensión de la experiencia formativa

Respecto a los motivos de elección de la carrera técnica actualmente cursada, en el instrumento aplicado en este estudio se ofreció una serie de alternativas de respuesta que incluían desde aspectos vocacionales hasta opciones prácticas de financiamiento o cercanía con el hogar.

A partir de esto, los argumentos que explican la elección de la carrera en la que actualmente se encuentran los estudiantes de CFT se resumen, básicamente, en dos perspectivas: (a) la selección de ésta a partir de la orientación vocacional o interés en el tema (la carrera que más me gusta), y (b) la selección a partir de una orientación racional en función de perspectivas de inserción laboral (elección debido a la existencia de un campo profesional atractivo). Aunque, por cierto, ambas motivos no son necesariamente contradictorios, estos dos tipos de respuesta predominan frente a motivos de continuidad temática con estudios anteriores, razones espurias (cercanía con el hogar, determinación de los padres) o ausencia de motivación particular en la elección de la carrera.

Considerando las respuestas desde una perspectiva de género, es posible observar una mayor recurrencia de respuestas masculinas en el campo de la racionalidad laboral, cuestión que varía en el caso de las mujeres, quienes se inclinan, mayormente, hacia razones de tipo vocacional. El gráfico siguiente sintetiza las respuestas: 
Gráfico n. ${ }^{\circ}$ 7. Motivos de elección de carrera estudiantes CFT por sexo.

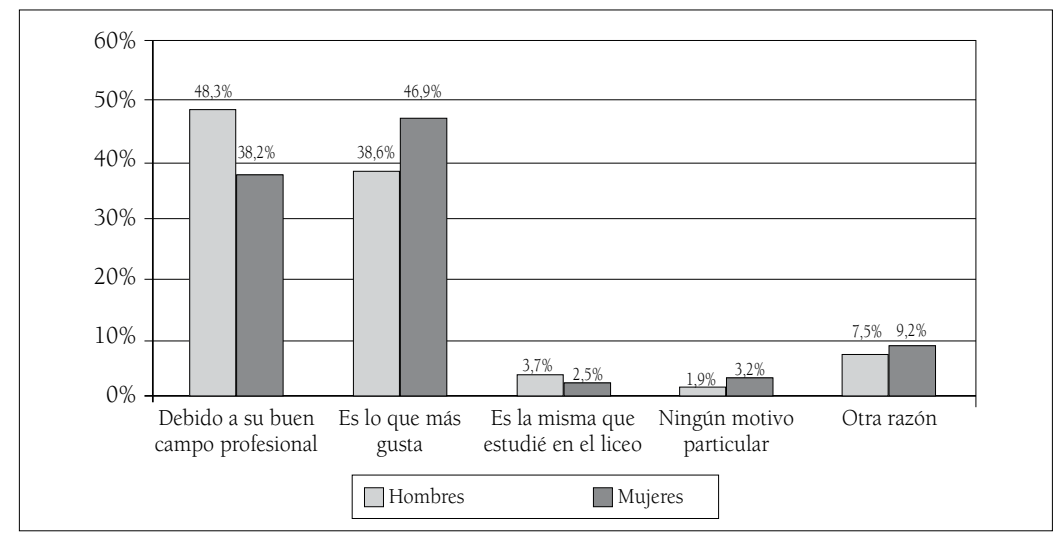

La variable edad también incide en la definición de las principales motivaciones en la elección de carrera; de este modo, mientras que el $45 \%$ de los estudiantes de 20 o menos años de edad selecciona la opción vocacional, ésta se reduce al 36\% de los casos de aquellos que tienen 30 años o más. La relación es inversamente proporcional a la elección debido a la consideración de su campo laboral; esto último podría explicarse en el caso de la población estudiantil de mayor edad, por el hecho de que resultaría mayormente recurrente la elección de un campo formativo en función de la actividad laboral desarrollada.

Respecto a la elección del CFT donde se realizan los estudios, las respuestas tienden a destacar el prestigio y buena calidad de la institución (21\% de las respuestas), o porque ese centro de estudios era la mejor alternativa para estudiar la carrera de interés personal (30\% de las respuestas). Con todo, llama la atención que un quinto de los encuestados en esta investigación señale que la elección del CFT fue condicionada por razones económicas: el 20\% de los estudiantes reconoce que el motivo principal por el que escogieron ese CFT era debido a que en este centro podían pagar el arancel. Este último hecho resulta relevante si se consideran algunos indicadores de diferenciación de ingresos de egresados del sistema de CFT. De acuerdo con los antecedentes disponibles, las carreras de mayores ingresos de nivel técnico presentan una alta dispersión de ingresos mensuales, lo que sugiere una distinción importante en el mercado 
laboral en relación con el centro de estudios donde los estudiantes se titularon (Meller, 2010).

Las condicionantes reseñadas evidencian las limitaciones de apoyo institucional para el financiamiento de los estudios de quienes ingresan a este sistema y, sobre esa base, la persistencia de un mercado de oferta formativa menos competitivo pero disponible para quienes presenten dificultades de financiar su formación de nivel superior. Como se sabe, desde sus inicios, la realización de estudios en una carrera de CFT ha estado asociada a las limitaciones institucionales de acceso a becas y créditos en comparación con las opciones existentes en el sistema universitario con financiamiento estatal; con todo, en los últimos años ha habido un incremento de alternativas de apoyo a los estudiantes que ingresan en este sistema. En este estudio se consultó a los encuestados acerca del acceso a algún beneficio que permitiese ayudar a sostener los costos de sus estudios. La información se resume en las siguientes tablas:

Tabla n. ${ }^{\circ}$ 2. Total de beneficiarios de becas para estudios en CFT.

\begin{tabular}{|l|r|r|r|c|}
\hline Beneficio becas & Hombre & Mujer & Total & $\%$ del Total \\
\hline Beca Presidente de la República & 3 & 7 & 10 & $1,1 \%$ \\
\hline Beca de Excelencia Académica & 3 & 11 & 14 & $1,5 \%$ \\
\hline Beca Nuevo Milenio & 84 & 156 & 240 & $26,6 \%$ \\
\hline Beca para Hijos de Profesionales de la Educación & 4 & 2 & 6 & $0,7 \%$ \\
\hline Otra beca estatal & 10 & 6 & 16 & $1,7 \%$ \\
\hline Otra beca no estatal & 11 & 20 & 31 & $3,4 \%$ \\
\hline Total & 115 & 202 & 317 & $35,2 \%$ \\
\hline
\end{tabular}

Tabla n. ${ }^{\circ}$ 3. Total de beneficiarios de crédito de financiamiento para estudios en CFT.

\begin{tabular}{|l|r|r|r|c|}
\hline Beneficio Crédito & Hombre & Mujer & Total & $\%$ del Total \\
\hline Crédito Universitario o Fondo Solidario & 0 & 3 & 3 & $0,3 \%$ \\
\hline Crédito CORFO & 2 & 0 & 2 & $0,2 \%$ \\
\hline Crédito con Aval del Estado & 49 & 91 & 140 & $15,5 \%$ \\
\hline Otro crédito otorgado por CFT & 19 & 21 & 40 & $4,4 \%$ \\
\hline Otro crédito & 11 & 5 & 16 & $1,7 \%$ \\
\hline Total & 81 & 120 & 201 & $22,3 \%$ \\
\hline
\end{tabular}

Como puede verse, reporta acceso a becas un máximo de 35\% de los estudiantes, mientras que un porcentaje menor, el 22,3\%, señala tener acceso a algún tipo de crédito para el financiamiento de 
sus estudios; el 56\% de los estudiantes no recibe ningún tipo de beca o crédito específico.

Los dos instrumentos más relevantes de acceso a beca o crédito estudiantil para quienes cursan sus estudios en un CFT son: (a) la Beca Nuevo Milenio (esta beca es destinada a estudiantes egresados de enseñanza media sin importar el año de egreso, y que se matriculen en primer año en una carrera conducente al título de Técnico de Nivel Superior en las Instituciones de Educación Superior Elegibles, que son determinadas por el Ministerio de Educación ${ }^{24}$ ), que es recibida por el 26,6\% de los estudiantes, y (b) el Crédito con Aval del Estado ${ }^{25}$, que es utilizado por el 15,5\% de los participantes en la encuesta.

Al revisarse el perfil de los estudiantes que reciben estos beneficios, como se observó en las tablas precedentes, hay una predominancia de mujeres que acceden tanto a becas como a crédito; de igual manera, al considerar las categorías de edad de los encuestados, existe una mucho mayor frecuencia de casos entre los estudiantes más jóvenes, decreciendo de manera considerable a partir de los 25 años de edad. En el caso del Crédito con Aval del Estado, la relación es relativamente similar.

Por otra parte, tan importante como lo anterior es el acceso a becas o créditos de los estudiantes de acuerdo con el centro de formación donde realizan sus estudios. El siguiente gráfico refleja las diferencias existentes en este plano según si se trata de un CFT autónomo o no.

24 La beca financia la totalidad o parte del arancel anual de la carrera, con un monto límite de $\$ 500.000$ (en el caso del año 2010); las exigencias para su otorgamiento son: pertenecer a los dos primeros quintiles de ingreso y tener un promedio de notas mínimo de 5.0 , si se pretende estudiar una carrera técnica de nivel CFT. La beca también apoya a estudiantes que se inscriban en carreras profesionales acreditadas al 31 de diciembre del año anterior e impartidas por institutos profesionales.

25 El sistema institucional de créditos para estudios superiores reporta la existencia de nueve centros de formación que destinan un poco más de 11.300 cupos para acceso a este tipo de créditos (el grueso de estos cupos son definidos por los CFT de mayor tamaño en el mercado de educación técnica del país). Las exigencias para acceso al crédito son en algunos casos puntaje de PSU y promedio de notas de la enseñanza media, mientras que en otros sólo se exige este último requisito. Como referencia general, el año 2008 fueron otorgados un total de 9.934 créditos a estudiantes de carreras técnicas (incluye universitarias), correspondientes al 23,3\% del total del sistema. Véase http://www.ingresa.cl/ 
Gráfico n. ${ }^{\circ}$ 8. Situación de acceso a beca y crédito por tipo de CFT (autónomo/no autónomo).

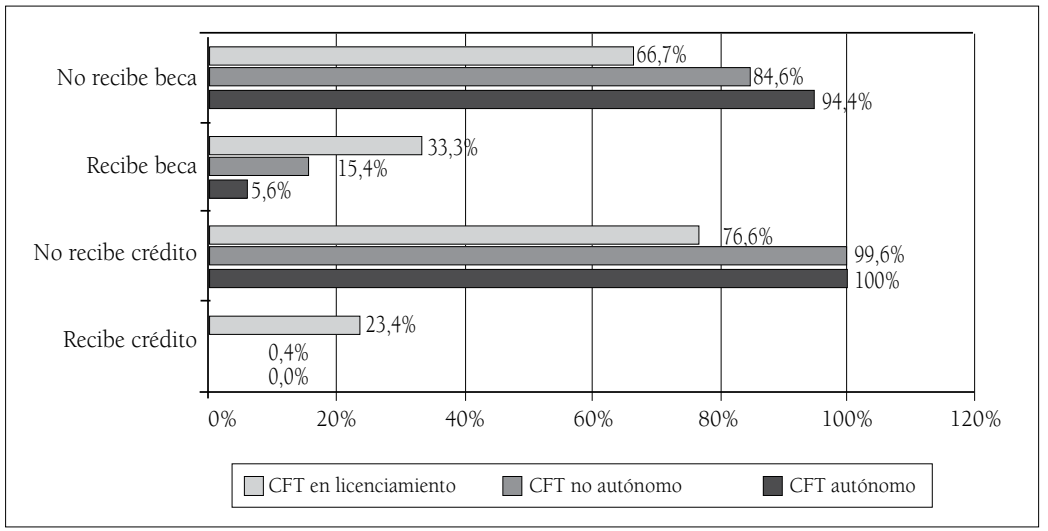

Como puede verse, un tercio de los encuestados que estudian en CFT autónomos recibe la Beca Nuevo Milenio, muy por encima del $15,4 \%$ y $5,6 \%$ de aquellos que lo hacen en centros de carácter no autónomo y en licenciamiento. En el caso del Crédito con Aval del Estado, la situación es más aguda, con predominio de los estudiantes de CFT autónomos entre quienes reciben este beneficio.

Aunque los datos recogidos señalan un avance relevante en el acceso a beneficios para el financiamiento de los estudios de quienes ingresan a la educación técnica superior, de todos modos, no puede obviarse el hecho de que un poco más de la mitad de los encuestados señala no disponer de suficiente información para postular a algún tipo de beneficio. De igual manera, los antecedentes indican que los instrumentos de apoyo institucional generados en el último tiempo se asocian, mayormente, a la figura de un estudiante que realiza un tipo de transición lineal. No es evidente que este tipo de instrumentos sean adecuados para quienes ingresan a estudiar una carrera desde una experiencia propiamente laboral.

(f) Proyecto de futuro: la extensión del periodo formativo como una realidad transversal

Respecto a la proyección de futuro una vez finalizado, los estudios de su actual carrera de CFT, cerca del 56\% de los encuestados manifiesta 
su interés de continuar estudios, ya sea en un instituto profesional o en una universidad; en términos generales esta manifestación de interés se sitúa dentro del área temática de su actual carrera. Por otra parte, alrededor de un $40 \%$ de los estudiantes señala que su propósito inmediato es trabajar, predominando la intención de hacerlo en el área de sus estudios actuales. Sorprendentemente, esta última opción recibe mayor apoyo entre los estudiantes de menor edad (grupo de 20 años o menos), casi 15 puntos por arriba incluso que el grupo de 30 años o más, tal como se observa en el siguiente gráfico:

Gráfico n. ${ }^{\circ}$ 9. Perspectivas de futuro al egreso de carrera CFT por edad.

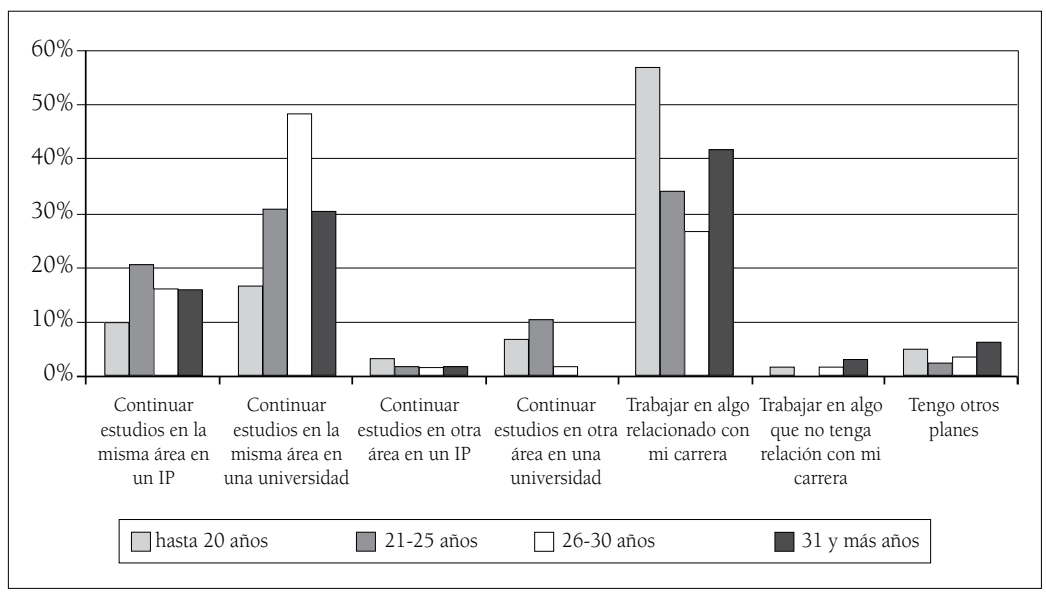

Lo anterior permite afirmar que el grueso de los estudiantes de carreras de CFT, sin que existan diferencias marcadas por grupo de edad, comparte un proyecto educativo, o una perspectiva de continuidad de su trayectoria educativa, que trasciende la duración de su actual carrera técnico-profesional.

Tal como se observa en el gráfico siguiente, cuando se interroga a los estudiantes acerca de lo que les gustaría estar haciendo en dos años más (es decir, un periodo de tiempo que cubre $1 \frac{1}{2} 2$ veces la duración de su actual carrera), el 55\% de las respuestas señala continuar estudiando. Esta perspectiva resulta por sobre el promedio en el grupo de edad de 26-30 años, mientras que más de la mitad de los estudiantes de 31 años y más también se ubica en esta posición. Las respuestas refuerzan la impresión de que la mayoría de los estudiantes 
de CFT comparte un modelo de transición educativo/laboral mucho más complejo que el modelo lineal tradicional, rompiendo las barreras de un límite de edad determinado.

Gráfico n. ${ }^{\circ}$ 10. Proyecto personal (estudio/trabajo) estudiantes CFT en dos años más (al momento de responder la encuesta).

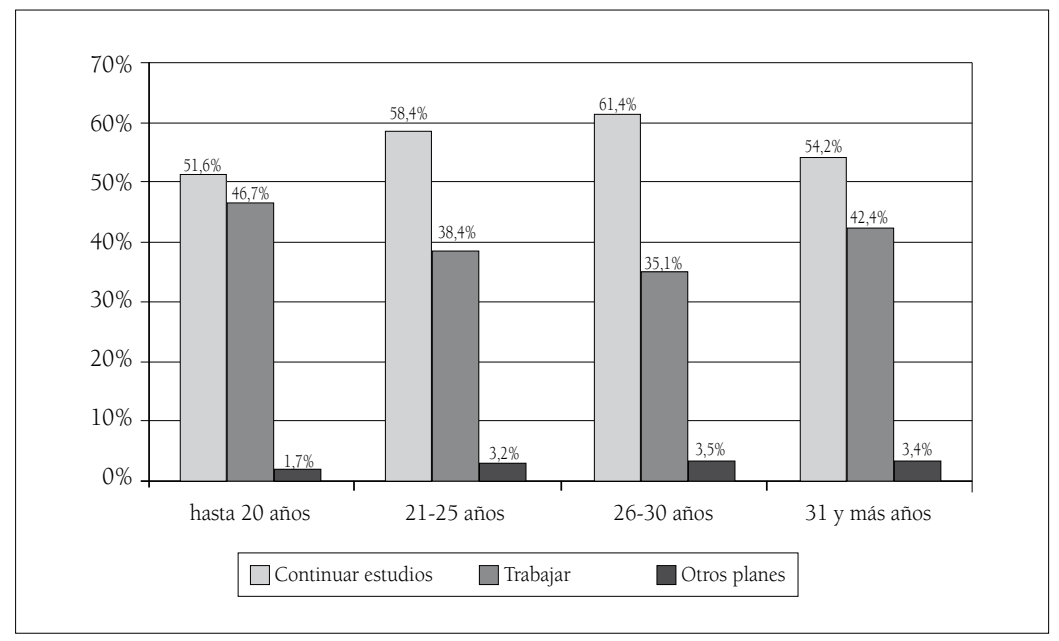

La continuidad de estudios tal como se refleja en las respuestas precedentes constituye un proyecto personal que, parece evidente, acentúa la necesidad de su articulación con actividades laborales. En efecto, para más del 70\% de quienes manifiestan el interés de continuar estudiando una vez finalizada su actual carrera, la única alternativa de financiamiento corresponde a los recursos generados por su propio trabajo, siendo un grupo menor el que señala la eventualidad de recurrir a becas o algún sistema de crédito. Lo anterior obliga, a la mayoría de los estudiantes, a acometer un esfuerzo de tipo individual para cubrir los costos involucrados en el proceso formativo; este hecho refuerza la imagen de una carrera individualizada y una trayectoria que se prolonga en el tiempo, perfil predominante entre los actuales estudiantes de carreras de CFT en nuestro país.

\section{Conclusiones}

A partir de los antecedentes recogidos en este estudio, se destacan las siguientes conclusiones: 
(a) En primer lugar, el estudio permite resaltar ciertas características particulares del perfil de los estudiantes que ingresan al sistema CFT, delimitando un rasgo específico, hasta ahora poco destacado en la caracterización de la demanda hacia este sistema formativo. En efecto, los resultados de la indagación señalan que solamente un segmento menor de quienes estudian una carrera técnica impartida por un CFT ha hecho una transición lineal de la educación media a esta modalidad formativa. A diferencia de lo anterior, el sistema se caracteriza por acoger a una mayoría de estudiantes que provienen de experiencias educativas y/o laborales diversas, y un promedio de edad elevado.

Existe una alta recurrencia de casos que inicia una carrera técnica después de un periodo prolongado de trabajo o de la combinación de actividades de estudio y desarrollo de una experiencia laboral. Lo anterior reafirma la multiplicidad de trayectorias posibles de los estudiantes del sistema y su heterogeneidad en consideración a la variable edad y experiencia educativo/laboral. Como se ha sostenido, esta evidencia permite indicar que un porcentaje importante de los actuales estudiantes de CFT, y por cierto, aquellos de mayor edad, ingresan a estudiar la carrera técnica en función de su actividad laboral actual, y no al revés, como sugiere un modelo de transición lineal, existiendo, de este modo, una racionalidad, mayormente asociada a la demanda de mercado y la propia experiencia laboral de un número importante de estos estudiantes.

(b) En la definición del perfil tipo del estudiante de carreras de CFT, también se observa una alta incidencia de personas que cursaron su educación media bajo la modalidad científico-humanista, no existiendo, por tanto, una secuencia lógica de estudios desde la modalidad técnico-profesional impartida en la enseñanza media; de igual manera, entre estos últimos, un porcentaje relevante ingresa a una carrera distinta al área de especialización de su formación inicial, relevando la debilidad de los itinerarios formativos que sugiere la distinción científico-humanista y técnico-profesional de nuestra enseñanza media. 
Al comparar los modelos de trayectoria de los actuales estudiantes de CFT en relación con sus estudios previos de enseñanza media, se observa una mayor incidencia de la experiencia laboral entre quienes egresaron de la enseñanza media técnico-profesional, EMTP. A diferencia de esto, la realización de estudios previos es una situación más recurrente entre los estudiantes de la enseñanza media chilena, $\mathrm{EMCH}$. En todo caso, en el estudio no se evidencian diferencias a partir de la situación de entrada de quienes cursan las respectivas carreras; en términos globales, la inserción en una carrera CFT responde más bien a un modelo fragmentado de trayectoria educativo/laboral, sin que se evidencien condiciones o exigencias previas para el ingreso a una carrera de este sistema.

(c) Junto a los aspectos antes reseñados, también se observa en el sistema CFT una clara segmentación de la matrícula a partir de la consideración de las variables de género y edad. Los antecedentes recogidos permiten destacar un predominio de mujeres jóvenes en las carreras de carácter diurno de este sistema. Como se señaló, es posible identificar dos modelos tipo de estudiantes de educación técnica de nivel superior, esto es, estudiantes más jóvenes y mayoritariamente mujeres en la modalidad diurna, y estudiantes de mayor edad y principalmente hombres bajo la modalidad vespertina.

Es factible sostener que estas características, incluso por encima del origen social de los estudiantes (considérese que, comparativamente, las universidades del Consejo de Rectores, CRUCH concentran una mayor proporción de estudiantes de los dos quintiles de más bajos ingresos en su matrícula), constituyen los rasgos distintivos del perfil de quienes cursan estudios bajo el sistema CFT. Por lo mismo, su consideración podría ser un factor de enriquecimiento de una política nacional de fortalecimiento de la educación técnica de nivel superior.

(d) En perspectivas de transición, el estudio arroja que un segmento importante de los actuales estudiantes de CFT señala su interés de continuar estudiando una vez finalizada su carrera. 
Esta disposición es transversal y también tiene una alta incidencia entre los estudiantes mayores de 25 años, por lo que, menos que tratarse de una actividad destinada a satisfacer una necesidad pragmática o reforzar competencias exigibles en el lugar de trabajo, el hecho de ser, actualmente, estudiante de una carrera de CFT parece estar asociado a la voluntad de construcción de una trayectoria educativa/laboral más larga, siendo éste un eslabón dentro de un proceso mayor.

Desde este punto de vista, los actuales estudiantes de CFT, por lo menos en su predisposición personal, se muestran alineados a la idea de una formación a lo largo de la vida y una alta tendencia a combinar actividades formativas y laborales en ese proceso.

(e) Finalmente, el análisis de trayectorias de los estudiantes de CFT refleja la debilidad de la organización institucional para favorecer itinerarios formativos coherentes en el nivel de la educación técnica, observándose, en términos globales, una fuerte desvinculación entre el sistema de formación de nivel medio y la oferta de nivel terciario, representada en este caso en el modelo de los centros de formación técnica. Lo anterior obliga a un debate sobre la pertinencia de fomentar puentes formativos entre la enseñanza secundaria y la oferta técnica de nivel superior. Los antecedentes de este estudio señalan que la persistencia de un sistema formativo de nivel técnico está mayormente asociado a decisiones personales y a la capacidad de agencia de los actores para trazar un itinerario formativo en esta área, abriendo la interrogante acerca de la pertinencia de políticas institucionales más robustas de articulación institucional en función de señales productivas de carácter transversal.

\section{Referencias bibliográficas}

Álamos, P. (2002) "La formación técnica superior en Chile"; Ministerio de Educación, MECESUP, Boletín n 3:4-14, Santiago.

Araneda, H. (2006) "Hacia un sistema de aprendizaje a lo largo de la vida: Oportunidad del sistema de certificación de competencias laborales" En Foco n. ${ }^{\circ} 75$; Expansiva, Santiago. 
Bernasconi, A. (2006) "Donde no somos tigres: problemas de la formación técnica en Chile en el contexto latinoamericano" En Foco n. ${ }^{\circ}$ 72; Expansiva, Santiago.

Comisión Asesora de Formación Técnico-Profesional (2009) "Bases para una política de formación técnico-profesional en Chile", Santiago.

Consejo Nacional de Innovación (2007) Estrategia Nacional de Innovación (enero 2007), Santiago. Disponible en: www.consejodeinnovacion.cl

De Moura, C. y García, C. Eds. (2003) "El modelo del instituto técnico superior norteamericano"; Banco Interamericano de Desarrollo, Washington.

Donoso, S. y Cancino, V. (2007) "Caracterización socioeconómica de los estudiantes de educación superior por tipo de institución"; CSE, Santiago.

Dubet, F. (2005, julio-diciembre) "Los Estudiantes" CPU-e; Revista de Investigación Educativa 1. Recuperado el 21.06.2010 de: http://www. uv.mx/cpuenuml/inves/estudiantes.htm\#

Du Bois-Raymond, M. y López, A. (2004) "Transiciones tipo yo-yo y trayectorias fallidas: hacia las políticas integradas de transición para los jóvenes europeos"; Revista de Estudios de Juventud n. ${ }^{\circ} 65$ / 1129; Madrid.

Edwards, A. (2005) "Cambios en el mercado laboral en América Latina y el Caribe: ¿Qué significa para el sector educativo?”; en Espínola, V. y García, N. "Modernización de la educación técnica postsecundaria: Opciones y desafíos para América Latina y el Caribe"; BID, Washington.

González, L.E. (1990) "La formación de técnicos superiores en los Centros de Formación Técnica"; en Lemaître, M.J. (Ed) "La educación superior en Chile: Un sistema en transición"; Colección Foro de Educación Superior, Corporación de Promoción Universitaria (CPU), Santiago.

Furlong, A. y Cartmel, F. (2009) "Mass higher education"; en Furlong, A. (ed) "Handbook of youth and young adulthood; new perspectives and agendas"; Routledge, UK.

Heinz, W. (2009) "Youth transitions in age of uncertainty"; en Furlong, A. (ed.) "Handbook of youth and young adulthood; new perspectives and agendas"; Routledge, UK.

Heinz, W. y Krüger, H. (2001) "Life course: innovations and challenges for social research"; en Current Sociology, vol. 49 (2), 29-45 SAGE Publications; London, UK.

Heinz, W. y Marshall, V. (2003) "Social Dynamics of the life course: transitions, institutions, and interrelations"; Aldine de Gruyter, New York. 
Hernández, L. y Paredes, R. (2007) "Restricciones económicas en la decisión de continuar estudios superiores técnicos o profesionales"; en Revista Calidad de la Educación n. ${ }^{\circ} 27$, pp. 238-261.

MINEDUC, Bases para una Política de Formación Técnico-Profesional en Chile, Informe Ejecutivo, Santiago 2009, en: http://www.formaciontecnica. cl/archivosNoticias/informe_bases.pdf

Larrañaga, O. (2006) "Costos y beneficios de estudiar en Centros de Formación Técnica: ¿Neutralidad de la política pública?”; Expansiva; Serie En Foco n. ${ }^{\circ} 71$, Santiago.

Meller, P. (2010) "Carreras Universitarias: rentabilidad, selectividad y discriminación”; Uqbar Ediciones y Centro de Investigación Avanzada en Educación (CIADE), Universidad de Chile, Santiago.

OCDE, Banco Mundial (2009) "Revisiones de políticas nacionales de educación: La educación superior en Chile".

Pérsico, P. y Pérsico, M. C. (1995) "Tendencias del Desarrollo de los Centros de Formación Técnica en Chile"; Corporación de Promoción Universitaria, CPU, Santiago.

Sánchez, S. y Escudero, C. (2008) "Trayectorias laborales y educacionales de los técnicos en Chile"; Informe Final en: http://www.redetis.org.ar/ media/document/trayectoriastecnicoschile.pdf

Secretaría Ejecutiva de Formación Técnico-Profesional, Comisión Asesora de Formación Técnico-Profesional: "Antecedentes y estrategia para la implementación de la política de formación técnico-profesional en Chile"; Santiago, 2010.

Sepúlveda, L. et al. (2009) "Estado y perspectivas de la enseñanza media técnico-profesional en Chile: un estudio sobre las orientaciones estratégicas predominantes en los actores"; Fondo de Investigación y Desarrollo en: Educación, FONIDE; Ministerio de Educación, Santiago. En http://w3app.mineduc.cl/mineduc/ded/documentos/ F310831_L_Sepulveda_UAH.pdf

Sistema Nacional de Información de la Educación Superior, División de Educación Superior, Ministerio de Educación: "Informe sobre retención de primer año de las carreras cohorte de ingreso 2007"; en: http:// www.divsuperior.cl/

Staff, J. y Mortimer, J. (2003) "Diverse transitions from school to work"; Work and Occupation; vol. 30, n. ${ }^{\circ}$ 3: 361-369.

Villarroel, K. /Secretaría Ejecutiva CNIC (2009) "Educación Superior en Chile: El informe OCDE / Banco Mundial a la luz de la estrategia de innovación"; en: http://biblioteca.cnic.cl/content/view/504445/ 
Educacion-Superior-en-Chile-El-informe-OCDE-Banco-Mundial-ala-luz-de-la-Estrategia-de-Innovacion.html

Wyn, J. y Dwyer, P. (2000) "Nuevas pautas en la transición de la juventud a la educación"; Revista Internacional de Ciencias Sociales n. ${ }^{\circ}$ 164:25, UNESCO.

Recibido: 05/07/2010

Aceptado: 11/10/2010 